\title{
Cost-effectiveness of adding psychomotor therapy to a multidisciplinary rehabilitation program for chronic pain
}

\author{
Lia C.C. van der Maas ${ }^{1-4}$, Judith E. Bosmans ${ }^{5}$, Maurits W. van Tulder ${ }^{5}$, Thomas W.J. Janssen ${ }^{3,4}$ \\ ${ }^{1}$ Human Movement and Education Division, University of Applied Sciences Windesheim, Zwolle - The Netherlands \\ ${ }^{2}$ Reade, Centre of Rehabilitation and Rheumatology, Amsterdam - The Netherlands \\ ${ }^{3}$ Reade, Amsterdam Rehabilitation Research Center, Amsterdam - The Netherlands \\ ${ }^{4}$ Amsterdam Movement Sciences, Faculty of Behavioural and Movement Sciences, Vrije Universiteit Amsterdam, Amsterdam - The Netherlands \\ ${ }^{5}$ Department of Health Sciences, Faculty of Science, Vrije Universiteit Amsterdam, Amsterdam Public Health Research Institute, \\ Amsterdam - The Netherlands
}

\begin{abstract}
Introduction: This study assesses the cost-effectiveness (CE) of a multidisciplinary pain rehabilitation program (treatment as usual [TAU]) with and without psychomotor therapy (PMT) for chronic pain patients.

Methods: Chronic pain patients were assigned to TAU + PMT or TAU using cluster randomization. Clinical outcomes measured were health-related quality of life (HRQOL), pain-related disability, and quality-adjusted life years (QALYs). Costs were measured from a societal perspective. Multiple imputation was used for missing data. Uncertainty surrounding incremental CE ratios was estimated using bootstrapping and presented in CE planes and CE acceptability curves.

Results: Ninety-four chronic pain patients ( $n=49$ TAU + PMT and $n=45$ TAU) were included. There were no significant differences in HRQOL, Pain Disability Index, and QALYs between TAU + PMT and TAU. Direct costs in TAU + PMT were significantly higher than in TAU (mean difference €3327, 95\% confidence interval [Cl] 1329; 5506). However, total societal costs in TAU + PMT were not significantly higher than in TAU (mean difference $€ 642,95 \% \mathrm{Cl}-3323 ;$ 4373). CE analyses showed that TAU + PMT was not cost-effective in comparison with TAU. Conclusions: Adding PMT to a multidisciplinary pain rehabilitation program is not considered cost-effective in comparison with a multidisciplinary pain rehabilitation program alone. The results of this study should be interpreted with caution because of the small sample size and high drop-out rate.
\end{abstract}

Keywords: Chronic pain, Cost-effectiveness, Multidisciplinary rehabilitation, Psychomotor therapy

\section{Introduction}

Chronic pain occurs in $19 \%$ of European adults and seriously impacts a patient's employment status, psychological health, and social well-being (1). Furthermore, the economic burden of chronic pain is considerable, with the major cost driver associated with productivity losses (2). In the Netherlands, it was estimated that approximately 10,000 patients per year receive a disability allowance because of

Received: February 12, 2020

Accepted: July 06, 2020

Published online: December 14, 2020

Corresponding author:

Lia C.C. van der Maas

PMT Amstelveen

Nijenburg 2F, 1081 GG Amsterdam- The Netherlands

lia@pmtamstelveen.nl chronic pain (3). The most prevalent body locations of chronic pain are lower back (21.3\%), shoulder (15.1\%), and neck $(14.3 \%)(4)$, which may explain why most cost-effectiveness (CE) studies among chronic pain patients evaluated interventions for chronic low back pain (5). The total societal costs of chronic back pain alone for the Dutch society were estimated at $€ 3.5$ billion in 2007 of which $88 \%$ was due to lost productivity costs (6). Although lost productivity costs are the largest cost driver, costs due to increased healthcare utilization are also enormous (7). In the USA, it was estimated that chronic pain generates healthcare costs in the range of 70 billion dollars per year due to visits to general practitioners and orthopedic specialists, medication, surgery, nondrug treatment (e.g., massage, physical therapy, acupuncture), and treatment at specialized pain clinics (8). These costs exceed the combined costs of treating patients with coronary artery disease, cancer, and AIDS (7).

Standard treatment in most rehabilitation centers in the Netherlands consists of multidisciplinary pain programs, based on the biopsychosocial model. This biopsychosocial model is a widely accepted model of the understanding of 
chronic pain (9), in which chronic pain is considered a complex and dynamic interaction between physiological, psychological, and social factors. The main goals of multidisciplinary treatment programs are to decrease disability due to chronic pain and improve health-related quality of life (HRQOL). Although these programs seem to have positive outcomes on HRQOL and disability in the short term, the effects are not always sustained in the long term (10) and the costs of these programs are high (8).

Adding a component aimed at developing body awareness might improve the clinical outcomes of multidisciplinary programs (11-17). Psychomotor therapy (PMT) is an experience-based treatment that incorporates body awareness as a primary target of intervention (18). By improving body awareness, patients learn to use body signals, other than just pain-related signals, to determine in what physical and mental state they are (19). Being aware of the relation between physical and mental states in different contexts may lead to a better understanding of this bodily information, thereby increasing confidence in one's body and oneself $(20,21)$, which in turn may increase HRQOL and decrease disability.

In a previous study, a randomized controlled trial (RCT) was performed to evaluate a multidisciplinary rehabilitation program with and without PMT (22). PMT was found to be an effective addition to a multidisciplinary program on depression, catastrophizing, and body awareness (22). The aim of the current study was to assess the CE of this multidisciplinary rehabilitation program with and without PMT for chronic pain patients.

\section{Methods}

\section{Design and participants}

This CE evaluation was conducted alongside an RCT with an intervention period of 3 months and a follow-up of 12 months, performed in the Netherlands between November 2007 and July 2011 (22). All patients were referred by general practitioners, pain specialists, or medical specialists for chronic pain treatment to Reade, Centre for Rehabilitation and Rheumatology in Amsterdam. Cluster randomization was used to assign groups of four to six patients to a multidisciplinary program without PMT (treatment as usual; TAU) or a multidisciplinary program with additional group PMT (TAU + PMT). A staff member not involved in the treatment performed randomization according to a "biased coin" design (23) for 20 treatment groups, 10 groups for each condition. The results of the biased coin procedure were put in 20 sequentially numbered sealed envelopes. The logistic planner of treatment opened a sealed envelope to know if the group would or would not receive PMT. Approval for this research was obtained from the Medical Ethics Review Committee of the VU University Medical Centre in Amsterdam.

\section{Intervention}

TAU consisted of a multidisciplinary rehabilitation program containing relaxation $(6 \times 1.5$ hours), graded activity $(33 \times$ 1 hour), rational-emotive therapy $(9 \times 1$ hour, $6 \times 1.5$ hours), occupational therapy ( $6 \times 1.5$ hours), chronic pain education $(3 \times 1.5$ hours), sports $(10 \times 1$ hour $)$, partner education $(3 \times 1.5$ hours), coaching ( $4 \times 1$ hour), and start and closing sessions ( 1 hour each). The aim of the treatment was to learn how to cope with the pain, not reducing the pain. This group treatment was offered 3 days per week for 12 weeks, with a total duration of 94 hours. Two follow-up group sessions (1.5 hours) were offered after 3 and 6 months.

PMT consisted of 10 group sessions of 1.5 hours in addition to the multidisciplinary rehabilitation program described above. PMT is a therapy based on experience of participants. Movement, body-oriented techniques, and verbal reflection on self-experiences are used to explore behaviors, feelings, and thoughts (18). In the PMT group we focused on two main topics: body experience, and interaction and communication. First, the patients learn to be aware of different physical sensations that increase body awareness, for example, exercises in which patients make contact with other people or with different materials. Second, patients learn to interpret signals without negative thoughts and concomitant feelings, which decreases catastrophizing, for example, focusing only on the internal body sensations and describing them in a more distant and objective manner and/or normalizing feelings that patients have in a specific context. Third, patients learn to use this information from the body to act accordingly, thereby increasing self-efficacy, for example, communicating about what you feel, your boundaries, and asking for help in miscellaneous exercises (24).

Patients in both treatment conditions were urged to stop all other treatments, except medication. Patients who were still working at the time treatment started were given the advice to take sick leave from work during the multidisciplinary treatment. This advice was given because 3 days of treatment on top of work would have cost too much energy, physically and mentally, for most of these patients, which might even provoke more pain complaints.

\section{Clinical outcome measures}

The primary clinical outcome measures were HRQOL and pain-related disability. HRQOL was measured by the RAND36 (Dutch version) (25). It comprises 36 items covering eight dimensions of health, which can be summarized into a physical summary score and a mental summary score. Both scores are linearly converted to a scale from 0 (poor health) to 100 (excellent health), with higher scores indicating higher quality of life.

Pain-related disability was measured by the Pain Disability Index (PDI) (26) (Dutch version by Pain Management and Research Centre, University Hospital Maastricht, 1999). PDI is a 7-item self-report measure of pain-related disability, rated on an 11-point scale. Higher scores indicate more painrelated disability.

The EuroQol (EQ-5D-3L) (27) was used to calculate quality-adjusted life years (QALYS). We used the Dutch tariff (28) to convert EQ-5D health states to utilities. Utilities reflect preferences for different health states anchored at 0 (death) and 1 (full health). QALYs were calculated by multiplying the utility of a health state by the time spent in this health state. Transitions between health states were linearly interpolated. 
The maximum number of QALYs to be experienced over 15 months is 1.25 , that is, the equivalent of 15 months (1.25 years) spent in full health.

\section{Cost measures}

Cost data were collected at baseline, posttreatment (12 weeks), and at 6, 9, and 15 months follow-up. Physician and therapist (complementary or allied healthcare) visits, medication use, medical aids (e.g., wheelchair, walking stick, neck collar, wrist brace, etc.), and absenteeism from paid work were measured using self-completed cost diaries.

Intervention costs were calculated using compliance information. When a patient was $100 \%$ compliant, intervention costs of TAU were 94 hours $\times € 110=€ 10,340$ and of TAU + PMT 109 hours $\times € 110=€ 11,990$. One hour of rehabilitation treatment was valued at $€ 110$ (29).

Costs of productivity losses were estimated using the friction cost method (FCM), which assumes that sick workers are replaced after a certain period of time (friction period used was 154 days) (29). Average productivity costs per working hour according to age and sex were used to estimate the costs of absenteeism (29). Healthcare utilization was valued using Dutch standard costs (29). Costs of medical aids and costs of visits to complementary therapists were based on prices from suppliers of medical aids and the therapists themselves, respectively. Medication was valued using prices of the Royal Dutch society for Pharmacy, the G-standard (Zindex, The Hague, The Netherlands, 2006). The index year for this study was 2009.

\section{Statistical analyses}

The economic evaluation was performed according to the intention-to-treat principle. Multiple imputation (MI) according to the Multivariate Imputation by Chained Equations (MICE) algorithm was used to impute missing cost and effect data with SPSS 20.0 (IBM Corporation). Using predictive mean matching (PMM), 20 imputed data sets were generated, each of which was analyzed separately. Effect and cost estimates from the 20 complete data sets were pooled using Rubin's rules (30). Seemingly unrelated regression was used to estimate cost and effect differences adjusted for covariates between the groups while accounting for potential correlation between costs and outcomes (31). The analyses were corrected for baseline differences between treatment groups in age, gender, pain duration, and pain diagnosis (neck, localized pain, and generalized pain). Incremental cost-effectiveness ratios (ICERs) were calculated by dividing the difference in total costs between TAU + PMT and TAU by the difference in clinical effects. Nonparametric bootstrapping with 5,000 replications was used to estimate $95 \%$ confidence intervals around cost differences and the uncertainty surrounding the ICER and cost-utility ratio (32). The bootstrapped cost-effect pairs were graphically represented on CE planes (33). Costeffectiveness acceptability (CEA) curves were also estimated, showing the probability that PMT + TAU is cost-effective compared to TAU for a range of ceiling ratios. The ceiling ratio is the amount of money society is willing to pay to gain one extra unit of effect (34).

\section{Results}

During the inclusion period, 114 patients were referred for group treatment, of whom 94 gave informed consent. Forty-five of these 94 were randomized to TAU and 49 to TAU + PMT. At baseline, there were statistically significant differences between the two treatment groups in age, gender, pain duration, and pain diagnosis (Tab. I). All effect and cost analyses were corrected for these baseline differences.

TABLE I - Baseline characteristics of the patients randomized to TAU and PMT

\begin{tabular}{lcc}
\hline & $\begin{array}{c}\text { TAU } \\
(\mathbf{n}=\mathbf{4 5})\end{array}$ & $\begin{array}{c}\text { TAU + PMT } \\
\text { ( } \mathbf{n}=\mathbf{4 9 )}\end{array}$ \\
\hline Women (\%) & 71.1 & 91.8 \\
\hline Age (mean, SD) & $45.4(11.1)$ & $38.6(11.1)$ \\
\hline Nationality (Dutch\%) & 73.3 & 81.6 \\
\hline Marital status (\%) & & \\
Married/living together & 44.5 & 49.0 \\
Single & 28.9 & 38.8 \\
Divorced & 13.3 & 4.1 \\
Other & 13.3 & 8.1 \\
\hline
\end{tabular}

\begin{tabular}{lcc}
\hline Education level* (\%) & & \\
Low & 17.8 & 20.4 \\
Middle & 42.2 & 30.6 \\
High & 37.7 & 49.0 \\
Unknown & 2.2 & 0 \\
\hline Paid work (yes\%) & 13.3 & 16.3 \\
\hline Pain diagnoses (\%) & & \\
Generalized pain** & 24.5 & 16.3 \\
Localized pain*** & 57.7 & 44.9 \\
Neck pain & 17.8 & 38.8 \\
\hline
\end{tabular}

\section{Pain duration (\%)}

\begin{tabular}{lcc}
3 to 12 months & 4.4 & 4.1 \\
1 to 2 years & 8.9 & 32.7 \\
2 to 5 years & 31.1 & 28.6 \\
$>5$ years & 55.6 & 34.7 \\
\hline $\begin{array}{l}\text { Pain intensity }{ }^{+} \text {(NRS 0-10) } \\
\text { (mean, SD) }\end{array}$ & $5.78(1.73)$ & $5.51(1.76)$ \\
\hline EuroQol utility (mean, SD) & $0.48(0.31)$ & $0.51(0.27)$ \\
\hline $\begin{array}{l}\text { RAND-36, mental } \\
\text { component (mean, SD) }\end{array}$ & $39.59(12.29)$ & $39.89(10.45)$ \\
$\begin{array}{l}\text { RAND-36, physical } \\
\text { component (mean, SD) }\end{array}$ & $31.51(7.17)$ & $32.42(6.72)$ \\
\hline Pain disability (mean, SD) & $40.48(9.89)$ & $40.36(8.81)$ \\
\hline
\end{tabular}

NRS = numeric rating scale; PMT = psychomotor therapy; SD - standard deviation; TAU = treatment as usual.

* Educational level is based on the Dutch school system, where low education is primary school and lower vocational education, middle is mediocre vocational education and higher secondary education, and high is higher vocational education and university.

**Including fibromyalgia.

$* * *$ Including (low) back pain, pain in upper extremities, and joint pain.

${ }^{+}$Average of least and usual pain. 
Complete cost and effect data were available for 18 (40\%) of the patients in the TAU group and 19 (39\%) of the patients in the TAU + PMT group. Reasons for loss to follow-up were unknown in 57\% (TAU 53\%; PMT 60\%) of the cases. Reasons that patients did mention were other priorities $(n=8)$, pregnancy $(n=5)$, relapse $(n=4)$, discontinued treatment $(n=3)$, and moved away $(n=1)$. At baseline, patients without complete cost and effect data had more pain, a lower score on the physical component of HRQOL, and were more frequently non-Dutch than patients with complete cost and effect data.

\section{Compliance with the intervention}

Twenty-four percent of the TAU patients were $100 \%$ compliant with the intervention. Mean compliance was 93\%, with a minimum of $54 \%$. Although none of the patients in TAU + PMT was $100 \%$ compliant, $33 \%$ of the patients was $100 \%$ compliant with PMT. Mean compliance in TAU + PMT was $92 \%$ with a range of $78 \%$ to $99 \%$.

\section{Clinical outcomes}

Clinical outcomes are presented in Table II. After 15 months, there were no statistically significant differences in PDI, in physical and mental components of HRQOL, and in QALYS.

TABLE II - Multiple imputed pooled clinical outcomes over 15 months

\begin{tabular}{|c|c|c|c|}
\hline $\begin{array}{l}\text { Outcome } \\
\text { measure }\end{array}$ & $\begin{array}{l}\text { TAU + PMT } \\
\text { Mean (SE) }\end{array}$ & $\begin{array}{c}\text { TAU } \\
\text { Mean (SE) }\end{array}$ & $\begin{array}{c}\text { Corrected } \\
\text { Difference } \\
\text { Mean }(95 \% \mathrm{Cl})\end{array}$ \\
\hline$\triangle$ RAND-36, PCS & $4.66(1.01)$ & $2.33(1.08)$ & $2.02(-1.42 ; 5.46)$ \\
\hline$\triangle$ RAND-36, MCS & 3.79 (1.59) & $4.98(1.85)$ & $-1.58(-6.32 ; 3.17)$ \\
\hline$\triangle \mathrm{PDI}$ & $-5.75(1.19)$ & $-7.77(1.68)$ & $-1.91(-6.07 ; 2.24)$ \\
\hline QALY & $0.69(0.04)$ & $0.68(0.04)$ & $-0.01(-0.12 ; 0.09)$ \\
\hline
\end{tabular}

$\mathrm{Cl}=$ confidence interval; $\mathrm{MCS}=$ mental component summary score of the RAND-36; PCS = physical component summary score of the RAND-36; $\mathrm{PDI}=$ Pain Disability Index; PMT = psychomotor therapy; $\mathrm{QALY}=$ qualityadjusted life year; $\mathrm{SE}=$ standard error; TAU = treatment as usual.

\section{Costs}

Table III shows that the difference in total societal costs between treatment groups (€642) was not very large in relation to the mean total societal costs in the two groups ( $€ 31,537$ for TAU and $€ 31,888$ for TAU + PMT). The main driver of the total societal costs was costs of absenteeism, which were $€ 2,685$ lower in TAU + PMT in comparison with those of TAU. However, this difference was not statistically significant $(95 \%$ confidence interval $[\mathrm{Cl}]-6175 ; 751)$. All healthcare costs were higher in the TAU + PMT group than in the TAU group, with statistically significant differences in medication costs (mean difference $€ 343,95 \% \mathrm{Cl} 183$; 626) and intervention costs (mean difference $€ 1,388,95 \% \mathrm{Cl} 1108 ; 1670$ ). This difference in intervention costs was due to the costs of additional PMT; 10 sessions of 1.5 hours results in 15 hours of rehabilitation treatment extra that costs $€ 110$ per hour, resulting in a total cost of adding PMT to the multidisciplinary treatment of $€ 1,650$.

TABLE III - Multiple imputed pooled costs over 15 months

\begin{tabular}{|c|c|c|c|}
\hline Costs & $\begin{array}{l}\text { TAU + PMT } \\
\text { Mean (SE) }\end{array}$ & $\begin{array}{c}\text { TAU } \\
\text { Mean (SE) }\end{array}$ & $\begin{array}{c}\text { Corrected } \\
\text { Difference } \\
\text { Mean }(95 \% \mathrm{Cl}) \\
\end{array}$ \\
\hline Absenteeism & 11802 (997) & 15396 (1950) & $-2685(-6175 ; 751)$ \\
\hline $\begin{array}{l}\text { Healthcare } \\
\text { utilization }\end{array}$ & 8077 (772) & 6517 (642) & $1590(-347 ; 3770)$ \\
\hline $\begin{array}{l}\text { Intervention } \\
\text { costs }\end{array}$ & 11065 (93) & $9637(136)$ & $1388(1108 ; 1670)$ \\
\hline Medical aids & $99(24)$ & $94(26)$ & $5(-72 ; 73)$ \\
\hline Medication & $494(78)$ & $243(50)$ & $343(183 ; 626)$ \\
\hline $\begin{array}{l}\text { Total healthcare } \\
\text { costs* }\end{array}$ & $19735(788)$ & $16492(661)$ & $3327(1329 ; 5506)$ \\
\hline $\begin{array}{l}\text { Total societal } \\
\text { costs }\end{array}$ & 31537 (1108) & 31888 (1946) & $642(-3323 ; 4373)$ \\
\hline
\end{tabular}

$\mathrm{Cl}=$ confidence interval; $\mathrm{PMT}=$ psychomotor therapy; $\mathrm{SE}=$ standard error; $\mathrm{TAU}=$ treatment as usual.

*Total healthcare costs $=$ direct costs.

\section{Cost-effectiveness}

The results of the CE and cost-utility analyses are presented in Table IV. The ICER for improvement in the physical component of HRQOL (RAND-36 physical component summary score of the RAND-36 [PCS]) at 15 months was 318, meaning that one point extra improvement on the RAND-36 PCS in the TAU + PMT group costs $€ 318$ more in comparison with the TAU group.

The CE plane (Fig. 1) shows that the majority of the costeffect pairs is located in the NE (TAU + PMT is more effective and more expensive than TAU) quadrant (57\%), which means that adding PMT to TAU was more effective but also more expensive. However, the CE plane also confirms that differences in costs and effects were not statistically significantly different between groups. The CEA curve in Figure 2 shows that at a ceiling ratio of $€ 0$ per point improvement on the physical component of HRQOL, the probability that TAU + PMT is costeffective in comparison with TAU was $37 \%$. At a ceiling ratio of $€ 1,000$ this probability increases to $70 \%$. At an even higher ceiling ratio of $€ 3,000$ or more this probability increased to approximately $83 \%$ (Fig. 2).

The difference in QALYs after 15 months between the treatment groups was very small, leading to a large ICER. The ICER indicates that a loss of $1 \mathrm{QALY}$ in the TAU + PMT group costs $€ 64,200$ more in comparison with the TAU group. At a ceiling ratio of $€ 0$ per point of improvement on the QALY, the probability that TAU + PMT is cost-effective in comparison with TAU was $37 \%$. At higher ceiling ratios this probability decreased a little to $34 \%$.

The ICER for PDI and the mental component of HRQOL suggested that adding PMT to the multidisciplinary program was more expensive and less effective than the multidisciplinary treatment alone. 
TABLE IV - Results of cost-effectiveness and cost-utility analyses

\begin{tabular}{|c|c|c|c|c|c|c|c|}
\hline & \multirow{2}{*}{$\begin{array}{l}\text { Cost difference } \\
\text { Mean }(95 \% \mathrm{Cl})\end{array}$} & \multirow{2}{*}{$\begin{array}{l}\text { Effect difference } \\
\text { Mean }(95 \% \mathrm{CI})\end{array}$} & \multirow[t]{2}{*}{ ICER } & \multicolumn{4}{|c|}{ Distribution CE plane } \\
\hline & & & & NE (\%) & SE (\%) & SW (\%) & NW (\%) \\
\hline \multicolumn{8}{|l|}{ Corrected* } \\
\hline RAND-36, PCS & $642(-3323 ; 4373)$ & $2.02(-1.42 ; 5.46)$ & 318 & 57 & 30 & 5 & 9 \\
\hline RAND-36, MCS & $642(-3323 ; 4373)$ & $-1.58(-6.32 ; 3.17)$ & -406 & 18 & 9 & 26 & 47 \\
\hline PDI & $642(-3323 ; 4373)$ & $-1.91(-6.07 ; 2.24)$ & -336 & 12 & 6 & 29 & 53 \\
\hline QALY & $642(-3323 ; 4373)$ & $-0.01(-0.12 ; 0.09)$ & -64200 & 26 & 12 & 22 & 39 \\
\hline
\end{tabular}

$\mathrm{Cl}$ = confidence interval; ICER = incremental cost-effectiveness ratio; $\mathrm{MCS}=$ mental component summary score of the RAND-36; NE = TAU + PMT is more effective and more expensive than TAU; NW = TAU + PMT is less effective and more expensive than TAU; PCS = physical component summary score of the RAND36; PDI = Pain Disability Index; PMT = psychomotor therapy; QALY = quality-adjusted life year; SE = TAU + PMT is more effective and less expensive than TAU; $\mathrm{SW}=\mathrm{TAU}+\mathrm{PMT}$ is less effective and less expensive than TAU; TAU = treatment as usual.

*Adjusted for baseline significant differences between groups on gender, age, pain duration, and pain diagnosis.

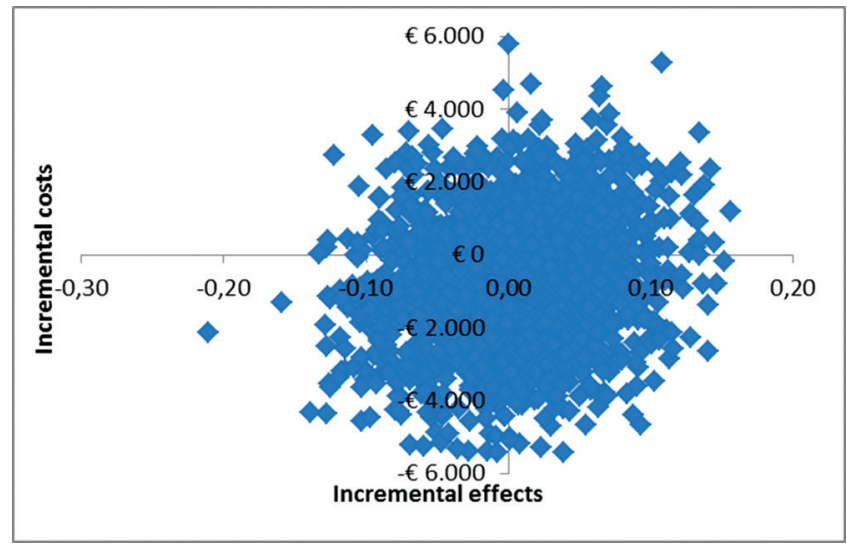

Fig. 1 - Cost-effectiveness (CE) plane of the physical component of health-related quality of life (HRQOL).

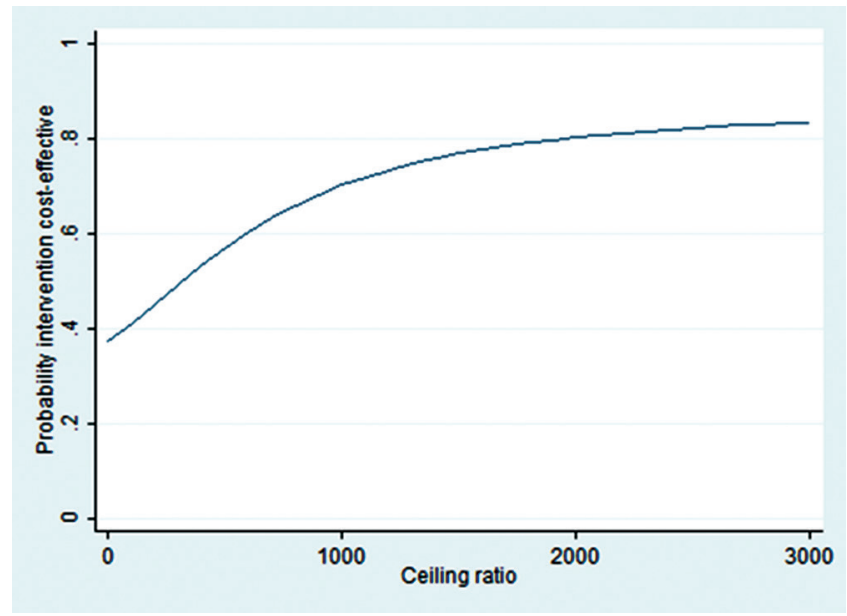

Fig. 2 - Cost-effectiveness acceptability curve of the physical component of health-related quality of life (HRQOL).

\section{Discussion}

The results show that there were no statistically significant differences in HRQOL and pain-related disability between TAU + PMT and TAU patients. Total societal costs were higher in the TAU + PMT group than in the TAU group, but this difference was not statistically significant. CE analyses showed that TAU + PMT was not cost-effective in comparison with TAU for QALY, PDI, and HRQOL.

Healthcare costs were higher in the TAU + PMT group than in the TAU group, partly because of the higher intervention costs, but also because medication costs were higher in the TAU + PMT group than in the TAU group. In the TAU + PMT group, more patients took more than three different types of medication, and medication was more expensive compared to the TAU group. This might suggest that patients in the TAU + PMT group experienced more severe complaints and complaints other than pain. However, pain intensity at baseline was not statistically significantly different between the groups. Also, at baseline, the number of patients that reported other complaints or disease was not statistically significantly different between the two treatment groups. The severity of these other complaints or diseases reported did not seem to show a clarifying difference either. It is possible that the differences in pain duration, pain diagnosis, age, and gender on baseline between TAU + PMT and TAU explain these medication differences. Often, first-line treatment of pain consists of a host of pharmacological agents (7). However, more than half of the patients taking medication felt that they were somewhat, not very, or not at all effective and $26 \%$ of the patients taking prescription medication stopped taking their medication (1). Therefore, a hypothesis could be that with shorter duration of pain, which was the case in the TAU + PMT condition, more medication is taken.

The observation in this study that absenteeism was the main driver of the total societal costs is in line with the few cost(-effectiveness) studies evaluating multidisciplinary rehabilitation programs for low back pain patients (2). In this study, absenteeism in the TAU + PMT group was lower than in the TAU group. This difference in absenteeism, although not 
statistically significant, might be explained by the fact that TAU + PMT patients improved more on the physical component of HRQOL than the TAU patients. Patients with improved physical functioning would be expected to return to work earlier (35).

To the best of the authors' knowledge, this was the first CE study of PMT as an added component to a multidisciplinary chronic pain rehabilitation program not specifically focused on a specific pain location (5). Therefore, comparison with other studies is difficult. Schweikert et al. (2) found no statistically significant difference in QALYs when adding just one component (cognitive behavioral treatment) to a multidisciplinary treatment for chronic low back pain, resulting in very large ICERs (€126,731/QALY). This was in line with our study. Furthermore, the study of Schweikert et al. (2) also showed that adding one component to a multidisciplinary chronic low back pain treatment did not result in significant differences between treatment conditions in HRQOL, which is in line with the findings of this study.

One of the strengths of this study is that it was a pragmatic trial from a societal perspective, meaning that it resembled daily clinical practice as much as possible and measured costs from a broad view that stretched beyond the impact on the individual patient. Another strength of this study is that patients were followed up for 15 months, which is a considerable duration and should be long enough to establish a change in clinical outcomes and costs. This is essential when evaluating multidisciplinary rehabilitation programs teaching patients to cope with the pain, because these interventions are costly and are expected to have an effect in the long term. The third strength is that the population in this study consisted of patients with different chronic pain locations, which corresponds better to clinical practice, where patients with different pain locations are treated together in one group. Finally, this study is one of the first that evaluated the CE of a single treatment component that was added onto a multidisciplinary rehabilitation program. To assess whether effectiveness of multidisciplinary treatment programs for chronic pain patients can be enhanced and number or length of sessions can be reduced by focusing on the most effective elements of the program, the relative contribution of each single treatment component of the multidisciplinary rehabilitation program must be known (2). Therefore, it is important that other single treatment components and different combinations of components of multidisciplinary pain rehabilitation programs are studied to find out which combination of treatment elements in a multidisciplinary pain rehabilitation program is most cost-effective.

Our study also had some limitations. First, the number of patients that did not return all cost questionnaires was considerable. The cost questionnaires may have been too burdening for most patients in this study who had to prioritize in their daily tasks because of their lower physical and mental capacity. There were some statistically significant differences between completers and non-completers on pain intensity, nationality, and the physical component of HRQOL, which suggested selective drop-out. We tried to overcome this limitation by using $\mathrm{Ml}$ for missing values. By including the variables on which completers and non-completers differed in the imputation model, we expect that the chance of bias caused by selective drop-out was reduced. Second, our study was underpowered to detect relevant cost differences, which is reflected in the wide confidence intervals around the cost differences. This is a common problem in economic evaluations alongside clinical trials. Because of the heavily skewed distribution of cost data, very large numbers of patients are needed to detect relevant cost differences (36). Third, randomization in the study was not completely successful, which resulted in statistically significant differences on age, gender, pain diagnosis, and pain duration between the two treatment conditions. In the analysis we corrected for these baseline differences. Fourth, in the RCT a statistically significant difference was found between the two treatment conditions on depression and catastrophizing in favor of TAU + PMT (22). However, in this CE study only the primary outcome variables $\mathrm{HRQOL}$ and disability were incorporated. Finally, the data used in this study is 11 years old. In these years TAU has changed on multiple points due to new insights (also gained from the results of this study), patients with different needs, and changes in financial flow. TAU is stretched out in a basic part of 17 weeks 2 days a week and when needed an additional period tailored to the patient's needs. The psychological intervention shifted from traditional cognitive behavioral therapy to acceptance and commitment therapy. Interventions directed to body awareness are now integrated in all different elements of TAU. And PMT became an optional intervention in the additional period after 17 weeks.

Based on the results of this study, adding PMT to a multidisciplinary rehabilitation program consisting of components covering physiological, psychological, and social areas is not considered cost-effective in comparison with this multidisciplinary rehabilitation program alone. The results of this study should be interpreted with caution because of small sample size and high drop-out. No strong recommendations for clinical practice can be given. Although multidisciplinary rehabilitation for chronic pain is considered beneficial for a variety of chronic pain problems, a substantial proportion of patients do not benefit at all. Therefore, it is important to investigate which components of multidisciplinary rehabilitation programs are effective and which combination of treatment elements is most effective and cost-effective.

\section{Disclosures}

Conflict of interest: The authors declare that there is no conflict of interest.

Financial support: This research received no specific grant from any funding agency in the public, commercial, or not-for-profit sectors.

\section{References}

1. Breivik H, Collett B, Ventafridda V, Cohen R, Gallacher D. Survey of chronic pain in Europe: prevalence, impact on daily life, and treatment. Eur J Pain. 2006;10(4):287-333.

2. Schweikert B, Jacobi E, Seitz R, et al. Effectiveness and costeffectiveness of adding a cognitive behavioral treatment to the rehabilitation of chronic low back pain. J Rheumatol. 2006; 33(12):2519-26. 
3. Lousberg R. Chronic pain, Multiaxial Diagnostics and Behavioral Mechanisms: University of Maastricht; 1994.

4. Picavet HSJ, Schouten JSAG. Musculoskeletal pain in the Netherlands: prevalences, consequences and risk groups, the DMC3-study. Pain. 2003;102:167-178.

5. Becker A. Health economics of interdisciplinary rehabilitation for chronic pain: does it support or invalidate the outcomes research of these programs? Curr Pain Headache Rep. 2012; 16(2):127-132.

6. Lambeek LC, van Tulder MW, Swinkels ICS, Koppes LLJ, Anema JR, van Mechelen W. The trend in total cost of back pain in the Netherlands in the period 2002 to 2007. Spine. 2011;36(13):1050-1058.

7. Turk DC. Clinical effectiveness and cost-effectiveness of treatments for patients with chronic pain. Clin J Pain. 2002;18(6): 355-365.

8. Gatchel RJ, Okifuji A. Evidence-based scientific data documenting the treatment and cost-effectiveness of comprehensive pain programs for chronic nonmalignant pain. J Pain. 2006;7(11):779-793.

9. Gatchel RJ, Peng YB, Peters ML, Fuchs PN, Turk DC. The biopsychosocial approach to chronic pain: scientific advances and future directions. Psychol Bull. 2007;133(4):581-624.

10. Hauser W, Bernardy K, Arnold B, Offenbacher M, Schiltenwolf M. Efficacy of multicomponent treatment in fibromyalgia syndrome: a meta-analysis of randomized controlled clinical trials. Arthritis Rheum. 2009;61(2):216-224.

11. Grahn EBM, Ekdahl CS, Borgquist L. Effects of a multidisciplinary rehabilitation programme on health-related quality of life in patients with prolonged musculoskeletal disorders: a 6-month follow-up of a prospective controlled study. Disabil Rehabil. 1998;20(8):285-297.

12. Haugli L, Steen E, Lærum E, Nygard R, Finset A. Learning to have less pain - is it possible? A one-year follow-up study of the effects of a personal construct group learning programme on patients with chronic musculoskeletal pain. Patient Educ Couns. 2001;45(2):111-118.

13. Kabat-Zinn J, Lipworth L, Burney R. The clinical use of mindfulness meditation for the self-regulation of chronic pain. J Behav Med. 1985;8(2):163-190.

14. Landsman-Dijkstra JJA, van Wijck R, Groothoff JW. The longterm lasting effectiveness on self-efficacy, attribution style, expression of emotions and quality of life of a body awareness program for chronic a-specific psychosomatic symptoms. Patient Educ Couns. 2006;60:66-79.

15. Malmgren-Olsson E, Armelius B, Armelius K. A comparative outcome study of body awareness therapy, feldenkrais, and conventional physiotherapy for patients with non-specific musculoskeletal disorders: changes in psychological symptoms, pain, and self-image. Physiother Theory Practice. 2001;17(2):77-96.

16. Mehling WE, Hamel KA, Acree M, Byl N, Hecht FM. Randomized, controlled trial of breath therapy for patients with chronic low-back pain. Altern Ther Health Med. 2005;11(4):44-52.

17. Gard G. Body awareness therapy for patients with fibromyalgia and chronic pain. Disabil Rehabil. 2005;27(12):725-728.

18. Probst M, Knapen J, Poot G, Vancampfort D. Psychomotor therapy and psychiatry: what's in the name? Open Compl Med J. 2010;2:105-113.
19. Steen E, Haugli L. From pain to self-awareness - a qualitative analysis of the significance of group participation for persons with chronic musculoskeletal pain. Patient Educ Counsel. 2001; 42(1):35-46.

20. Haugli L, Steen E, Laerum E, Finset A, Nygaard R. Agency orientation and chronic musculoskeletal pain: effects of a group learning program based on the personal construct theory. Clin J Pain. 2000;16(4):281-289.

21. Landsman-Dijkstra JJA, van Wijck R, Groothoff JW, Rispens $P$. The short-term effects of a body awareness program: better self-management of health problems for individuals with chronic a-specific psychosomatic symptoms. Patient Educ Counsel. 2004;55:155-167.

22. Van der Maas LC, Köke A, Pont M, et al. Improving the multidisciplinary treatment of chronic pain by stimulating body awareness: a cluster-randomized trial. Clin J Pain. 2015 Jul;31(7): 660-669.

23. Efron B. Forcing a sequential experiment to be balanced. Biometrika. 1971;58(3):403-417.

24. Van der Meijden, van der Kolk H, Bosscher R. Psychomotorische Therapie voor mensen met chronische pijn; een methodisch raamwerk. Zwolle: Christelijke Hogeschool Windesheim, 2007.

25. Van der Zee KI, Sanderman R, Heyink JW, de Haes H. Psychometric qualities of the RAND 36-item health survey 1.0: a multidimensional measure of general health status. Int J Behav Med. 1996;3(2):104-122.

26. Pollard CA. Preliminary validity study of the Pain Disability Index. Percept Mot Skills. 1984;59(3):974.

27. EuroQoL Group. EuroQol - a new facility for the measurement of health-related quality of life. Health Policy. 1990;16:199-208.

28. Lamers LM, Stalmeier PF, McDonnell J, Krabbe PFM, Busschbach JJ. Kwaliteit van leven meten in economische evaluaties: het Nederlands EQ-5D-tarief. Nederlands Tijdschrift voor Geneeskunde. 2005;149:1574-1578.

29. Hakkaart-van Roijen L, Tan SS, Bouwmans CAM. Handleiding voor kostenonderzoek, methoden en standaard kostprijzen voor economische evaluaties in de gezondheidszorg. College voor zorgverzekeringen; 2011.

30. Rubin DB. Multiple imputation for nonresponse in surveys. New York: Wiley; 1987.

31. Willan AR, Briggs AH, Hoch JS. Regression methods for covariate adjustment and subgroup analysis for non-censored costeffectiveness data. Health Econ. 2004;13(5):461-475.

32. Efron B. Missing data, imputation, and the bootstrap. J Am Stat Assoc. 1994;89(426):463-75.

33. Black WC. The CE plane - a graphic representation of cost-effectiveness. Med Decis Making. 1990;10(3):212-214.

34. Fenwick E, O'Brien BJ, Briggs A. Cost-effectiveness acceptability curves - facts, fallacies and frequently asked questions. Health Econ. 2004;13(5):405-415.

35. Schaafsma FG, Whelan K, van der Beek AJ, van der Es-Lambeek LC, Ojajarvi A, Verbeek JH. Physical conditioning as part of a return to work strategy to reduce sickness absence for workers with back pain. Cochrane Database Syst Rev. 2013;8:CD001822.

36. Briggs A. Economic evaluation and clinical trials: size matters the need for greater power in cost analyses poses an ethical dilemma. Br Med J. 2000;321(7273):1362-1363. 